\title{
PIEZAS OWENIANAS EN EL ROMPECABEZAS DARWINIANO
}

\author{
Daniel Blanco \\ UNL, CEHIF-UNQ, UNTREF \\ Email: danielblanco.fb@gmail.com \\ ORCID iD: https://orcid.org/0000-0001-9748-7000 \\ Santiago Ginnobili \\ CEFHIC-UNQ-CONICET, UBA, UNTREF \\ Email: santi75@gmail.com \\ ORCID iD: https://orcid.org/0000-0001-5375-965X
}

Recibido: 27 mayo 2019; Aceptado: 5 febrero 2020

Cómo citar este artículo/Citation: Blanco, Daniel; Ginnobili, Santiago (2020) "Piezas owenianas en el rompecabezas darwiniano", Asclepio, 72(2): p325. https://doi.org/10.3989/asclepio.2020.26.

RESUMEN: En este trabajo discutimos la extensión de la influencia que el pensamiento de Richard Owen tuvo sobre el de Charles Darwin. Además, se intentará mostrar lo heterogéneo de tal influencia, que va desde teorías específicas a giros retóricos. Esta influencia es en muchos casos subestimada, dando la sensación de que la novedad darwiniana consistió únicamente en mirar con ojos desprejuiciados lo que los otros no habían visto. Esta visión resulta injusta con Owen, y también con el esfuerzo conceptual llevado adelante por Darwin con las piezas brindadas por sus precursores. Finalmente, este es un caso interesante para entender el tipo de novedad aportada por las revoluciones científicas y el modo sofisticado en que tal novedad se sustenta sobre el trabajo de los enfoques previos.

PALABRAS CLAVE: Richard Owen; Charles Darwin; homologías; revolución científica; revolución darwiniana; precursor.

\section{OWENIAN PIECES IN THE DARWINIAN PUZZLE}

ABSTRACT: In this paper we discuss the extent of influence that Richard Owen's thinking had on Charles Darwin's thinking. In addition, we will try to show the heterogeneousness of this influence, which goes from specific theories to rhetorical turns. This influence is in many cases underestimated, giving the impression that the Darwinian novelty consisted solely in looking with unprejudiced eyes at what others had not seen. This vision is unfair to Owen, and to the conceptual effort carried out by Darwin with the pieces offered by his predecessors. Finally, this is an interesting case to understand the kind of novelty brought by scientific revolutions and the sophisticated way in which such novelty is based on the work of previous approaches.

KEY WORDS: Richard Owen; Charles Darwin; Homologies; Scientific Revolution; Darwinian Revolution; Precursor. 


\section{INTRODUCCIÓN}

La revolución darwiniana es probablemente el caso de revisión más abrupto de los registrados en la historia de la ciencia. Una vez concluida (en la medida en que pueda admitirse que concluyó), el mundo predarwiniano se volvió extraño, de una racionalidad difícil de inteligir. Otra característica peculiar de esta revolución es que se piensa alrededor de un único héroe. Esto se debe a que casi todos los elementos que configurarían el "nuevo mundo" fueron presentados de manera extensa y convincente en un único libro: El origen de las especies (OS en adelante). El resto de los guerreros, incluso quien codescubrió su teoría más famosa, aceptaron su subordinación al autodenominarse "darwinianos".

El calibre de esta revolución lleva a pensar que el genio de Darwin consistió en mirar de modo desprejuiciado aquello que a sus pares permanecía oculto, probablemente porque la pesada herencia obnubilaba su visión. Como su maestro Lyell, que había intuido que la erosión suave y el tiempo extenso podrían configurar cañones cuya enormidad parecían implicar catástrofes, Darwin había notado que leves variaciones en ese tiempo profundo podrían configurar lo que por su enorme complejidad sólo parecía poder haber surgido por un diseño preconcebido y deliberado. El "cómo no se me ocurrió a mí" de Huxley va en la misma doble dirección: el darwinismo sería un producto único que habría surgido espontáneamente en la mente de su creador.

Aunque Thomas Kuhn nos advierte de no caer como historiadores de la ciencia en el maniqueísmo de las historias oficiales que se tejen como legitimadoras de las teorías científicas triunfantes, su presentación general de la naturaleza de las revoluciones en ciencia (aunque no así los trabajos en los que aplica su enfoque a casos específicos) suele caracterizarse como revisiones disruptivas que abandonan vanos esfuerzos pasados.

Sin embargo, concediendo que existen diversos tipos de inconmensurabilidad entre paradigmas sucesivos, y que eso lleva a que no exista ningún modo objetivo de compararlos, si la revisión fuese absoluta, no habría modo alguno (local o subjetivo) de comparación, lo cual dificultaría entender cómo es que alguna revolución es siquiera posible. Kuhn (1970) tiene este punto en claro, y autores posteriores como Larry Laudan (1984) lo enriquecieron al mostrar cómo las revoluciones nunca revisan todo, utilizando lo no re- visado como plataforma móvil, frágil e intersubjetiva, desde la cual la discusión es posible.

Este punto es especialmente relevante para nuestro caso. Pues si queremos entender el genio de Darwin, la imagen del científico despertando con un "eureka" con lo fundamental del paradigma por venir, sencillamente no es adecuada. No lo es, porque no se condice con el lento esfuerzo plasmado en correspondencias y notas; y no lo es, porque omite la importancia de los enfoques previos que Darwin decide desarrollar. No tomar en cuenta esto implica no sólo ser injustos con los gigantes sobre cuyos hombros Darwin se asienta, sino que, además, nos lleva a una imagen falsa de su propio método y de la naturaleza de su genialidad.

Por otro lado, el héroe que crea ex nihilo no es uno que genere escuela, uno que sirva de modelo a seguir. La tarea de Darwin, en cambio, constituye un hermoso ejemplo para todos quienes quieran pensar original, osada y críticamente.

En realidad, Darwin construye su edificio bebiendo de fuentes diversas. En esta contribución trataremos una de ellas: la de Richard Owen, que probará ser especialmente interesante por varias razones:

En primer término, porque la historia ha sido especialmente injusta con él (Darwin incluido), probablemente debido a que se convirtió en opositor al darwinismo.

En segundo lugar, porque a pesar de ser reconocido como un enemigo del nuevo programa, Owen fue en algún sentido limitado, pero genuino, su precursor; y nos hemos propuesto aquí explicitar en qué sentido heterogéneo es que decimos tal cosa.

En tercer lugar, este estudio mostrará lo complejo que resulta discutir las influencias de parte de convicciones previas. Owen es un autor predarwiniano, que habita el mundo platónico de arquetipos, cadena del ser, diseño, etc. La reinterpretación que realiza Darwin sobre sus ideas es extrema, porque inaugura una nueva mirada; y a la vez, mínima, porque se aprovecha y mucho del trabajo realizado por Owen (y otros) casi sin modificación.

Colaborar con entender la extensión de la influencia de Owen sobre Darwin, y con la elucidación de su naturaleza, es el objetivo de nuestro trabajo. Concretamente, mostraremos cuánto del contenido conceptual del paradigma reemplazado -siempre acotándonos a las contribuciones de Owen- se encuentra 
heredado en el contenido conceptual del paradigma evolucionista reemplazante. A partir de esta conmensurabilidad parcial, reflexionamos acerca de la naturaleza de esta revolución científica particular confiando en que tal cosa permitirá entender mejor el modo en que se construyen los nuevos paradigmas.

El escrito está organizado del siguiente modo. En primer término (sección 2), comentamos brevemente el posicionamiento de Owen y Darwin respecto del debate forma-función.

Luego, en segundo lugar, pasamos a explicitar la deuda de Darwin para con Owen, tratando de elucidar su significación metateórica. Veremos cuánto de las obras de este último (prominentemente, Owen, 1843; 1847 ; 1849) es utilizado/reciclado por parte de Darwin en $O S$, incluso en aquellos aspectos teóricos en los que se diferencian. Tematizamos aquí influencias diversas relativas a: teorías subyacentes (sección 3), estrategias ofensivas (sección 4), argumentos explicativos (sección 5), el ordenamiento de la biodiversidad (sección 6), las fuerzas actuantes en la naturaleza (sección 7) y cuestiones programáticas (sección 8).

Finalmente, ofrecemos nuestras conclusiones.

\section{OWEN Y DARWIN FRENTE AL CONFLICTO FORMA Y FUNCIÓN}

El debate entre forma y función que permeó a la Europa continental durante varias décadas antes de la aparición de $O S$, tuvo su versión inglesa en el choque entre dos líneas de investigación. Una, con asiento en Oxford y Cambridge, acentuaba la relevancia del funcionalismo a la hora de pensar la biodiversidad; y otra, en Edimburgo, que hacía lo propio con el formalismo. Ambos enfoques, aparentemente irreconciliables, contribuyeron empero con un suelo fértil para el florecimiento del creacionismo en dos sentidos (Rupke, 2009b).

Por un lado, el énfasis en la estructura común de los organismos llevó a pensar que había un solo arquitecto detrás de la creación, y no muchos. Es aquí, y en deuda con autores continentales tales como Johann von Göethe, Carl Carus, Lorenz Oken, Félix Vicq d'Azyr, Geoffroy Saint-Hilaire y Étienne Serres, donde encontramos la enorme obra de Richard Owen ${ }^{1}$. Owen, justamente ( $y$ apartándose en esto de sus precursores), afirmó, en primer lugar, que el encuentro de la estructura constante en el grupo Ver- tebrata revela que existe "una sola causa de toda organización” (Owen, 1849, p. 38; 1859, p. 62), un creador único. En segundo término, Owen propuso que el plan base del creador era susceptible de ser descubierto científicamente merced a un nuevo programa en anatomía comparada (Owen, 1849, p. 75). En tercer lugar, Owen pretendió haber encontrado dicho plan en el arquetipo, un modelo -abstracto, según su propia adjetivación (Owen, 1849, p. 82)- a partir de cuya modificación, pérdida o enriquecimiento de partes se moldearon todas las formas del grupo.

Por otro lado, el enfoque funcionalista y su irrestricta búsqueda de "oficios" para virtualmente cada rasgo, fortaleció la confianza en la teología natural (cuéntese aquí los textos de John Ray, William Paley, o los Bridgewater Treatises) que infería a partir del ajuste entre rasgo y ambiente, el diseño de un constructor. La existencia y protagonismo de un creador inteligente se concluye a partir de la innegable adecuación de los organismos a sus respectivos roles en la naturaleza. Owen también adhería a esta postura (entonces un tanto relegadas), pero con dos mayúsculas notas al pie: (1) el origen de no todo rasgo habría de explicarse por razones funcionales, y, (2) el origen de rasgos que sí son funcionales (adaptaciones) han de explicarse por causas secundarias: el creador "previó" las distintas formas, pero no las creó individualmente ex nihilo de manera directa. En parte es por eso que, a su tiempo, a Owen no lo escandalizarán ni en el transformismo ni el naturalismo de Darwin.

Ambos creacionismos son compatibles: un anteproyecto divino es perfectamente integrable con el argumento del diseño (Rupke, 1993, pp. 248-249; 2009b; Bowler 2013), ya sea en la forma de creación especial o por causas secundarias. Como veremos, durante la década de 1840, Owen intentó justamente ligar el formalismo con el funcionalismo.

La revolución darwinista vino a cambiarlo todo en muchos sentidos. Acotándonos sólo al ámbito que venimos comentando, nos interesa subrayar:

(1) Darwin ofreció dos explicaciones científicas para ambos fenómenos: la teoría de la selección natural explicará la emergencia de las estructuras y sus roles en la economía de la vida, desplazando la teología natural derivada de la creación especial (que estrictamente no era, reiteramos, lo que Owen defendía); y la teoría del origen en común explicará la presencia 
de estructuras compartidas que reflejan un patrón común distinguible en la biodiversidad, desplazando la teoría arquetípica de Owen.

(2) En lo que puede considerarse uno de los esfuerzos más notables de unificación en la historia de la ciencia, Darwin logró mostrar lo que Owen sólo pudo vislumbrar: que las dos parcialidades del debate no eran excluyentes ahora fuera del marco creacionista. Por el contrario, ambos enfoques eran perfectamente armonizables, y podrían/debían aceptarse como facetas distintas, pero igualmente legítimas de la biología. Todos los rasgos organísmicos, forma y función, similitudes y diferencias, son resultados de una historia evolutiva, enlazándose así las dos posturas antes interpretadas como en conflicto.

En tanto que el objetivo compartido era acercar posiciones en la discusión dicotómica más importante de la época (ver sección 8); y en tanto Owen se ocupó del tema antes que Darwin, es que resulta deseable evaluar en qué sentido puede defenderse que aquel es precursor de este. Fuera de las interpretaciones de los historiadores, los precursores del darwinismo son listados por el propio Darwin en el Historical Sketch que incluyera en OS a partir de 1861. Allí aparece, entre otros, el nombre de Owen, aunque involucrado en un relato que Darwin fue cambiando en sucesivas ediciones. En las secciones que siguen esperamos mostrar en qué medida el número y la profundidad de tales influencias han sido mayor e injustamente desmerecidas por muchos, en cierta medida, como veremos, Darwin incluido.

\section{TEORÍAS SUBYACENTES}

El modo más contundente de señalar influencias sobre un autor es mostrar su aceptación de teorías previas. Sin embargo, en las discusiones de corte kuhniano, esto resulta difícil de identificar tal vez por la renuencia de los enfoques historicistas a dar cuenta de la estructura de las teorías. Para empeorar las cosas, el mismo Darwin no se esfuerza en distinguir entre las diferentes teorías que sostiene. Esto es bastante usual: muchas veces sucede que las teorías utilizadas en la práctica científica nunca son explicitadas, operando como conocimiento tácito, al modo en que lo hacen las reglas gramaticales de un lenguaje. Esto suele ocurrir especialmente con teorías presupuestas en la determinación de los fenómenos de los que se quiere dar cuenta: se vuelven parte del arsenal implícito con el cual el científico categoriza lo que ha de explicar; y que son caracterizados, ingenuamente, como "hechos" (Blanco, 2011).

La primera influencia (que es también la más notable) que Owen tuvo sobre Darwin es de este tipo. Darwin acepta sin modificación alguna las teorías owenianas que permiten detectar homologías, concretamente, lo que Owen denomina "homologías especiales" ${ }^{\prime 2}$. Esta conexión es la más discutida en el ámbito metateórico, prominentemente por la relevancia que tiene para juzgar la acusación contra la teoría del origen en común de incurrir en circularidad explicativa, cuestión heredada también en discusiones contemporáneas acerca de la cladística (cf. Rupke, 2009b; Pearson, 2010; Roffé et al., 2018; Roffé, 2020).

El punto es que el modo en que Owen caracteriza las homologías -que, como esperaríamos, no hace mención alguna al monofiletismo- se ve heredado sin modificación en Darwin (Blanco, 2012). Por el contrario, estas homologías no-históricas constituyen una conceptualización que Darwin incorporó en sus extensos estudios anatómicos de los cirrípedos (Darwin, 1851); en 1859, escribió de ellas aproblemáticamente, eran los "hechos" a explicar; y, en la última edición del libro, aprobó la inclusión de un glosario escrito por William Dallas donde aparecen descritas (cf. Darwin, 1872, pp. 383-386; Wood, 1995; Griffith, 2007; Padian, 2007).

Para el diagnóstico de homologías, Owen utiliza mayormente la receta de Saint-Hilaire que referencia una constancia en la posición relativa y conexión entre las partes de un rasgo (Owen, 1847, p. 6, 143; Aboitiz, 1988; Russell, 1916) $)^{3}$. Este protocolo de reconocimiento constituye una arista heurística para una "anatomía comparada homológica", y Owen propone una estandarización terminológica destinada a reflejar el reconocimiento de los mismos huesos en toda la serie Vertebrata.

Darwin (y en gran medida también los sistemáticos del presente) debe la determinación de homologías a los criterios operativos sistematizados por Owen, disponibles con anterioridad a 1859, y de los que se apropia acríticamente, asumiéndolos como fenómenos del mundo que los usuarios de la teoría del origen en común pretenderán explicar. De este modo, Darwin coincide con Owen tanto en que hay unidad en la diversidad, como en la manera en que hemos de proceder para detectarla 4 . 


\section{ESTRATEGIAS OFENSIVAS}

Darwin también apela a estrategias argumentativas owenianas (resignificándolas) cuando se ocupa de negar ciertas explicaciones para la presencia de homologías.

Owen menciona tres alternativas (Owen, 1849, p. 40):

(1) Aparecieron por casualidad.

(2) Aparecieron para cumplir funciones adaptativas compartidas (principios cuvieranos).

(3) Son manifestaciones de un tipo superior de conformidad estructural orgánica sobre la cual "ha complacido al creador enmarcar algunas de sus criaturas vivientes".

Owen no se toma siquiera una línea para refutar al azar, la primera alternativa.

Sobre la segunda alternativa, la explicación funcionalista, la juzga insatisfactoria. Como justificación, esgrime el caso de los múltiples puntos de osificación en el cráneo del feto humano. En principio, el funcionalista podría argumentar que la osificación tardía facilita el trabajo de parto para la madre. Pero esta explicación es inapropiada porque la situación se replica en las aves (donde no hay función paralela para la osificación vinculada con la rotura del huevo); en los pequeños embriones de marsupiales (donde el parto no es riesgoso para la madre); y también en cocodrilos y muchos peces. La hipótesis funcionalista simplemente no da cuenta de estas concordancias homológicas (Owen, 1847 , p. 73; 1849, pp. 9, 39-40).

Owen va más lejos. Ingeniosamente, concluye que la unidad de tipo denuncia la inadecuación de la entonces muy empleada analogía entre el diseño natural y el diseño humano (Paley, 1802): cuando construimos un dispositivo con cierta funcionalidad en mente desde cero, por separado de otras construcciones, no estamos atados al modo en que se hicieron otros aparatos. Por eso es que, dice Owen, no hay conexión entre un bote y un globo (Owen, 1849, pp. 9-10, 84-85). Si el diseño natural fuera como el humano, y por lo tanto "el único principio que gobierna la organización" fuera el funcional, no habríamos de esperar encontrar conformidad en las distintas adaptaciones a distintos fines. Sin embargo, en la naturaleza sí hay una constricción: la construcción de un ala, un remo, o un brazo se efectúa con las mismas porciones óseas, con las misma "materia prima" básica. Luego, la acción del creador no es analogable a la acción de un arquitecto humano.
Descartada la explicación funcionalista, Owen se inclina por la tercera alternativa, la explicación estructural. Concluye que la "hipótesis más probable" para explicar la unidad que revela la anatomía comparada es que existe una ley general de conformidad respecto del arquetipo vertebrado, el (único) plan básico predeterminado en la mente del (único) creador. Como comentamos en la introducción, Owen cree que el creacionismo está todavía a salvo si suponemos que el creador se basó en un plan original para derivar la biodiversidad. Sin embargo, nótese que una vez que se acepta la unidad de tipo, la teleología experimenta una subordinación con el estructuralismo impensable en autores como George Cuvier o Paley.

Darwin, por su parte, concuerda con Owen sobre las primeras dos alternativas, y lo hace citándolo. El azar es insatisfactorio, como también lo es el funcionalismo (Darwin, 1872, p. 384). Alude incluso a exactamente el mismo ejemplo que introdujera Owen para descartar la explicación adaptativa: las suturas craneales en organismos que no nacen a través de un canal de parto estrecho (Darwin, 1859, p. 197).

Aunque Darwin sigue al pie de la letra a Owen en lo que niega: ni el azar ni el funcionalismo pueden explicar estas similitudes, utiliza la estrategia argumentativa en dirección no de los arquetipos sino del origen común (ver sección 5.1). Ron Amundson nota incluso algo más: si bien hacia 1844 Darwin había visto que la unidad de tipo podía ser explicada por ancestría en común, probablemente sea gracias a Owen que asumiera la incapacidad de dar cuenta de ésta adaptativamente (Amundson 2007; Appel, 1987). El argumento es tan poderoso que se sigue usando todavía hoy, aunque usualmente el referenciado sea Darwin, y no Owen (Jacob, 1977; Gould, 1978, 1981; Dawkins, 2006).

Señalemos la magnitud de la incomunicación que puede existir en discusiones entre enfoques opuestos en un período revolucionario. Inconmensurabilidad mediante, no sólo ocurre que científicos pertenecientes a distintas tradiciones apelan a diferentes conjuntos de argumentos en su beneficio. En este caso, el mismo tipo de argumentos que para Darwin apuntaba hacía ancestros terrenales e imperfectos, apuntaban para Owen a la mente divina. Veremos en la sección siguiente, sin embargo, que las semejanzas importantes se encuentran no sólo a nivel de teorías subyacentes y/o a nivel de estrategias argumentativas, como hemos visto hasta aquí, sino también a nivel de las explicaciones ofrecidas para dar cuenta de la unidad de tipo. 


\section{INFLUENCIAS A NIVEL EXPLICATIVO}

\subsection{LA CONEXIÓN ENTRE ARQUETIPOS Y ANCESTROS}

Aunque coinciden en lo que niegan, Darwin y Owen defienden explicaciones distintas. Sin embargo, advertiremos que a pesar de la disparidad queda el resabio indisimulable de otra notable coincidencia, difícil de caracterizar metateóricamente.

Primeramente, Darwin objeta la alternativa creacionista de Owen por razones de cientificidad, y lo hace utilizando explícitamente sus palabras:

La perspectiva ordinaria de la creación independiente de cada ser, sólo podemos decir que así son las cosas; que le ha complacido al Creador construir a todos los animales y las plantas en cada clase abarcante en base a un plan uniforme; pero esta no es una explicación científica. (Darwin, 1872, p. 383, énfasis nuestro) $)^{5}$.

Debe haber, entonces, una cuarta alternativa que Owen no advirtió.

David Hull (1973) y Amundson (1998) coinciden en que mientras se considere al plan (el esquema arquetípico abstracto) como un pensamiento generado en la mente de un creador, podría considerarse que se dispone de una explicación. Ahora bien, sacar a Dios de la escena no implica renegar del plan. Ahora el plan, más que constituir una explicación, es un fenómeno que demanda de una, algo que Owen no provee en términos aceptables para Darwin.

La explicación de Darwin es, claro está, la ancestría en común y la consecuente preservación hereditaria. Es en esto que damos con la concurrencia: Darwin, con la teoría de comunidad de origen, no hace más (ni menos) que "corporizar" lo que Owen idealizó (hay algunos matices sobre esto que serán introducidos luego). El arquetipo de Owen es ahora un ancestro remoto de todos los organismos que comparten el plan Vertebrata. En el margen de su copia de On the Nature of Limbs, Darwin escribió:

Veo a los arquetipos de Owen como algo más que ideales, los veo como una representación real en tanto que una habilidad consumada y una generalización elevada pueden representar la forma parental de los vertebrados. (Fide Ospovat, 1981, p. 146)

$Y$ en OS:

Si suponemos que el progenitor ancestral, el arquetipo, como se lo ha llamado, de todos los mamíferos, poseía sus miembros construidos sobre un patrón general existente, para cualquier propósito al que pudieran servir, podemos percibir rápidamente el pleno significado de la construcción homóloga de los miembros a través de la clase entera. (Darwin, 1872, p. 384)

Darwin reemplaza el plan anatómico abstracto de Owen por un ancestro real, de "carne y hueso", conservando el resto de las intuiciones, incluyendo el término "arquetipo". En resumen, el conjunto de homologías en Vertebrata preserva su modo de determinación y extensión, con independencia de que sus elementos remitan a una figura arquetípica en la mente del creador o a un ancestro en común.

\subsection{LA "FORMA BÁSICA" Y EL LUGAR DE LOS HUMANOS}

El idealismo de Owen $(1849$, p. 2) ha sido cuestionado por el hecho que su arquetipo, a diferencia, p.e., del de Joseph Maclise, es mínimo, elemental, incipiente, y no completo y acabado como requeriría un platonismo auténtico (Rupke 1993, pp. 235, 243). La diversificación se obtiene más por modificación y agregado de partes, que por sustracción. No se trata de la suma de todos los endoesqueletos de la naturaleza, sino de un patrón básico compartido. Mientras que Maclise vincula al arquetipo con el esqueleto humano (el que supone el más completo y de mayor organización, que es lo que correspondería a un platonista genuino, el ejemplar más perfecto), Owen lo aparta de él.

Poco tiene en común la noción de comunidad de origen darwiniana con un arquetipo de corte platónico como el de Maclise, en donde el arquetipo es la versión más plena y perfecta. En cambio, su relación con el arquetipo oweniano, aunque compleja, es mucho más cercana.

Al igual que Owen, Darwin sostiene que el ancestro común remoto en casos de grupos amplios (como Vertebrata) debió haber sido extremadamente simple (Darwin, 1859, pp. 330-331). Cuanto más complicado el esqueleto, más diferenciado estará de dicho antecesor.

Además, ambos admiten la complejización, agregado, y pérdida de partes, por lo que ninguno sostiene el aumento de complejidad como flecha del tiempo. La intuición de Darwin es la de Owen, sólo que libe- 
rada de todo platonismo. Tanto el individuo remoto como la noción arquetípica unifican al mismo grupo de idéntica amplitud. La coincidencia está en la caracterización de simplicidad tanto del esbozo general como del ancestro primigenio.

Por otra parte, el modo en que Owen incluye a los humanos en la escena no debe desdeñarse. Éstos, en tanto que organismos extremadamente especializados, están en el ápice de la diversidad (Owen, 1843, p. 28; 1849, pp. 68-69), pero no son especiales en el sentido que fueran construidos siguiendo un plan individual distintivo (ver Owen 1857, pp. 19-20; 1859; cf. Gross, 1993a; 1993b). Por eso es que Owen insiste en que la anatomía comparada del grupo Vertebrata habría de ser útil a la medicina (Owen, 1843, p. 5; 1849, p. 118). Aprendemos acerca de los humanos estudiando al pez, pues en ambos es detectable el mismo arquetipo, el mismo plan básico (Owen, 1849, pp. 110-119). En este contexto, Owen se preocupa en mostrar el cariz empírico de su programa anatómico: rastrear el arquetipo desde el hombre al pez no es "un mero sueño trascendental, sino un conocimiento genuino y el fruto legítimo de la investigación inductiva" (Owen, 1849, p. 70).

Darwin, por supuesto, pondría a los humanos como parte del mundo animal (relativizando sus privilegios), aunque inicialmente apenas haya aludido a las consecuencias que conllevaría esta inclusión en su propia esfera transformista (Darwin, 1859, pp. 488). En definitiva, ambos coincidirían en que la anatomía comparada tiene resultados prácticos para la medicina humana.

\subsection{PRIMITIVISMO DE LA "FORMA BÁSICA"}

Owen señala que cuanto más primitiva la especie, mayor será su cercanía al arquetipo y menor su sofisticación (Owen, 1847, pp. 16, 117, 120; 1849, 18, 49-50, 59). Esto queda plasmado en su conocida representación del arquetipo de 1849 (ver Figura 1), el cual recuerda al por entonces recientemente descrito cefalocordado Amphioxus lanceolatus (Goodsir, 1844; Rupke, 1993; Padian, 2007) ${ }^{6}$.

Dicho grabado refleja la herencia en Owen de la teoría de la vértebra de Oken y Goethe (Owen, 1849, p. 41, 50-51; cf. Amundson, 2005, pp. 55-56). El arquetipo está compuesto por una serie de vértebras segmentadas desde el sacro al cráneo, y, ya sea el espécimen un pez, un reptil, un ave o un mamífero, remite a él (ver sección 8.1). Esta composición secuencial del arquetipo es lo que Owen llama "homología general". En On the Nature of Limbs, Owen presenta las modificaciones típicas del esqueleto de cada grupo, mostrando que el arquetipo es siempre rastreable, incluso "bajo todas sus modificaciones teleológicas". Pero, otra vez, a mayor primitivismo, menor modificación respecto del bosquejo original, es decir, más claras las huellas de las retenciones esenciales (Owen, 1849, p. 2).

Figura 1. El arquetipo de Owen según aparece en On the Nature of Limbs.

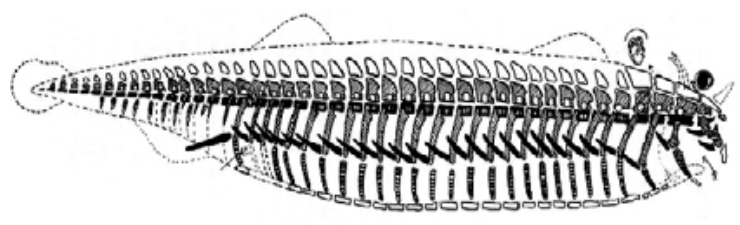

Aunque no se manifiesta respecto de la teoría de la vértebra, Darwin concordará, como decíamos, en que las especies más primitivas nos ayudan a vislumbrar el aspecto del ancestro primitivo de todos los vertebrados. A mayor simplicidad, normalmente (definitivamente no siempre) más troncal será su posición en el árbol de la vida, localización que lo vinculará con formas muy divergentes entre sí (cf. Darwin, 1859, pp. 330-331).

\section{FORMALISMO Y SISTEMÁTICA}

\subsection{LA CONSTRUCCIÓN DE UNA SISTEMÁTICA NATURAL}

Existen tres acuerdos principales entre Darwin y el formalismo de Owen respecto de la sistemática. En primer lugar, Darwin se pone del lado de Owen en la cuestión general de que los sistemáticos deberían atenerse a aspectos estructurales, más que funcionales, a la hora de efectivizar su tarea, enfrentándose así a coro a Cuvier, una discusión que, como vimos en la sección 2, fuera de enorme relevancia en la época (Appel, 1987; Russell 1916; Asma 1996; Ochoa y Barahona 2009).

En segundo término, coinciden en el doble objetivo de la sistemática: ordenar, sí, pero también plasmar con ese ordenamiento algo significativo, algo "real". Owen, al igual que Saint-Hilaire (y también Cuvier), 
estaba convencido de que los esfuerzos en anatomía serían de enorme utilidad para la construcción de una sistemática verdadera, esto es, una que no fuera un mero catálogo del caos de la biodiversidad, sino, desde una impronta realista, también un reflejo de algo que efectivamente está "ahí afuera". La taxonomía debía revelar un orden natural, y no uno arbitrario según criterios artificiales forjados por cuestiones pragmáticas específicas cambiantes. Independientemente de la discusión que existe respecto de la noción de especie en Darwin, éste afirma que "las clasificaciones no son un agrupamiento arbitrario como sucede con las estrellas en las constelaciones" (Darwin, 1859, p. 411), aceptando este realismo taxonómico.

Por supuesto, el cambio de arquetipos por ancestros involucra una importante novedad. Lo que está "ahí afuera" que la taxonomía vendría a mapear son relaciones parentales. Bajo la impronta distintiva del monofiletismo, Darwin dirá que "toda clasificación verdadera es genealógica” (Darwin, 1859, p. 400); es decir, una clasificación genuina, "natural", es la que reproduce los grados de parentescos que unifican todas las formas en un único árbol (o unos pocos árboles) de ascendencia en común.

Por último, en tercer lugar, Darwin se suma a Owen (1847, p. 139) en sostener el mismo criterio clave como guía para la clasificación: el encuentro de homologías desde la anatomía comparada (y la embriología y la histología), en oposición con el funcionalismo de Cuvier. El resultado constituirá un esquema taxonómico que revele el distanciamiento que sus poseedores tienen respecto de un origen/plan compartido. Que la explicación esgrimida para ese orden sea genealógica (Darwin) o trascendentalista (Owen) es irrelevante respecto de la posibilidad de organizar la biodiversidad bajo el mismo criterio que, al menos en principio, y como veremos en la siguiente sección, determinará un ordenamiento coincidente.

\subsection{Cantidad de tipos en la naturaleza}

No sólo hay acuerdo respecto del modo de ejecución en sistemática, sino que además no existen discrepancias sustantivas respecto de los resultados de dicho ordenamiento. Owen (1843, p. 12) pensaba que había cuatro grupos identificables en la naturaleza, y aunque se concentró en el tipo ideal para los organismos con endoesqueletos, también anticipó que tal vez una tarea análoga podría efectuarse en los otros grupos. Mantener estos tipos separados lo diferencia de Saint-Hilaire, de aceptar una cadena del ser sin hiatos, y/o de una concepción evolucionista que involucre a toda la vida. En 1843 escribió:

¡Cuán vasto el hiato que separa al insecto del pez ápodo, el cangrejo de la tortuga, y el insecto volador del ave o el murciélago! No existe un ascenso regular e ininterrumpido en la escala de la organización; ninguna continua cadena del ser. [...] Ni siquiera con la idea que ahora tenemos de las formas vivientes que poblaron este planeta durante épocas pasadas remotas de su historia, se pueden completar todas las brechas que existen y conectar juntas en una serie linear los miembros existentes y extintas del reino animal. (Owen, 1843, p. 267, 268)

Esta declaración no debe leerse como una oposición al cambio orgánico como un todo. Por el contrario, a esta fecha (Richards, 1987), Owen está plenamente dispuesto a aceptar cierto transmutacionismo, tal vez no lejos de lo que pronto publicaría Robert Chambers. De hecho, años más tarde, Owen $(1849$, p. 56) sostiene que hubo un tiempo en la Tierra que los peces eran los únicos representantes del grupo, y que cambios subsecuentes en las condiciones de existencia provocaron modificaciones organísmicas. Owen no niega vínculos entre miembros de uno y el mismo grupo, aunque sí respecto de vínculos entre los grupos. La anatomía del pez puede enseñarnos de la de los humanos, pero no así la de los invertebrados en tanto que pertenecen a otro grupo de conexión inexistente con los humanos (Owen, 1843, p. 361).

Por último, que Owen escriba precisamente de cuatro tipos (Owen, 1849, p. 87), es legado de Cuvier y de von Baer, quienes reconocen ese número exacto de formas: Vertebrata, Molusca, Articulata y Radiata ${ }^{7}$.

Darwin, a su vez, preserva el número:

No puedo dudar de que la teoría de la descendencia con modificación abarca a todos los miembros de una misma Clase. Creo que los animales han descendido de al menos cuatro o cinco progenitores, y que las plantas lo hicieron a partir de un número igual o inferior. La analogía me llevaría un paso más allá, esto es, a creer que todos los animales y las plantas han descendido de un único prototipo. Pero la analogía puede ser una guía engañosa. [...] Debería inferir por analogía que probablemente todos los seres orgánicos que han vivido en esta tierra han descendido de 
una forma primordial, en la que la vida fue insuflada por primera vez. (Darwin, 1859, pp. 483-484, énfasis nuestro)

Darwin veía difícil la unidad entre estos grupos, pero ensaya de todos modos la idea de un ancestro en común muy antiguo para todos los organismos ${ }^{8}$. Para Owen, tal unidad es simplemente impensable, aunque admite la posibilidad de hacer un trabajo análogo al que él hizo con Vertebrata, en el seno de los otros tres grupos. Después de todo, consideraba a su grupo preferido como la clase más diversificada (Owen, 1849, p. 52), por lo que si ha podido reconocer unidad de tipo allí, bien podría hacerse lo mismo en otros.

\section{FUERZAS ANTAGÓNICAS EN LA NATURALEZA}

\subsection{CONSERVACIÓN Y DIVERSIFICACIÓN DE LAS FORMAS}

El origen en común y la selección natural son antagónicas en un sentido análogo al advertido por Owen entre la fuerza conservadora ("polarizadora", "vegetativa") que retiene los rasgos arquetípicos, y la fuerza adaptativa que diversifica generando rasgos adaptados ("fitness", Owen, 1849, p. 38) a distintas funciones. Estas dos fuerzas son las responsables, respectivamente, de la unidad y de la diversidad entre las especies (Amundson, 2007).

Dice Owen:

La idea platónica de un principio organizador específico o fuerza parecería estar en antagonismo con la fuerza general polarizadora, y la supera y la moldea en subordinación a las exigencias de la forma específica resultante. (Owen, 1847, p. 172)

Así, la fuerza adaptativa vence a la conservativa torciendo la homogeneidad. ¿Por qué? Porque el instrumento debe estar en armonía con su oficio (Owen, 1849, p. 9), y, sin embargo, la modificación resultante no es lo suficientemente fuerte como para desdibujar completamente la unidad de tipo. Esto garantiza la promesa heurística de su propio programa (Owen, 1849, pp. 44, 64): los cambios podrán ser muchos, pero el anatomista avezado siempre podrá diagnosticar estar frente al mismo rasgo en distintas formas según convenga a la función de turno.

Como se ve, Owen adopta -todavía vagamente- una suerte de evolucionismo adaptacionista, esto es, guia- do por una organización especificadora y funcionalista. Parece sugerir implícitamente que las especies, construidas todas en base al plan arquetípico (en On the Nature of Limbs, directamente es el modelo primigenio en la mente planificadora divina), han sido modificadas en direcciones adaptativas por una energía/fuerza de la naturaleza que procede de los individuos mismos. Tengamos en cuenta que la sucesión de faunas en el registro fósil había sido aceptada a comienzos del siglo XIX, y que, como hemos dicho, Owen (1849, pp. 56, 60-62) explícitamente dice que hubo un tiempo en que los únicos vertebrados existentes eran los peces. Aceptar ambas cosas, naturalmente, no involucra una adhesión al transformismo (Owen, 1849, pp. 55-63), pero el subsecuente papel diversificador que ocupa el adaptacionismo en su pensamiento ciertamente no está en conformidad con el fijismo.

Igualmente, para Darwin, las distintas formas son resultado de esas mismas dos fuerzas en operación: la unidad de tipo, y la adaptación. Ambas son contempladas por sus dos teorías más importantes: la ancestría en común explica las semejanzas estructurales, y la selección natural esculpe adaptaciones en esas estructuras, explicando así la diversificación adaptativa. La contraposición entre ambas fuerzas, aunque no su explicación, se lee claramente en Owen.

Dos diferencias importantes pueden subrayarse aquí:

(1) Para Darwin, la fuerza adaptativa viene "desde afuera", desde la exigencia ambiental, mientras que Owen la piensa como una fuerza inherente a los organismos.

(2) Para Darwin, la fuerza adaptativa es todavía más fuerte que la fuerza conservadora; más de lo que Owen estuvo dispuesto a admitir.

Con todo, más allá del poder disolvente relativo que pueda tener el adaptacionismo, ambos autores coinciden en lo que éste logra/intenta: velar los indicios de la existencia de un plan común/comunidad de origen. En este sentido, Darwin está defendiendo algo bastante similar a lo que sostenía Owen, lo cual nos lleva al siguiente punto de contacto.

\subsection{EL PAPEL DESORIENTADOR DEL ADAPTACIONISMO}

Owen advierte que las fuerzas adaptativas generan estructuras que, en su modificación, borran las pistas arquetípicas que contribuyen a identificar homologías: 
[Las homologías] son progresivamente anuladas y enmascaradas por el acaecimiento de la ley superior de las modificaciones y adaptaciones especiales a las exigencias y hábitos y esfera de la vida de la especie. (Owen, 1847, p. 106)

Sin embargo, como anticipamos, las modificaciones a lo largo de la serie natural de los animales altamente organizados no se apartan completamente del tipo fundamental a pesar de la adaptación de las especies a sus diferentes oficios en la creación:

Hasta qué nivel las operaciones de la fuerza polarizante está sujeta a la organización de una forma animal específica se convierte en el signo de tales especies, y en el lugar en el que asciende en la escala del ser. Los lineamientos del arquetipo común son oscurecidos en el mismo grado. Pero incluso en el hombre, donde las fuerzas organizadoras específicas han ejercido sus poderes más altos, las características arquetípicas son rastreables. (Owen, 1847, p. 172).

Notemos otra vez su lejanía de un platonismo genuino: el hombre es ejemplo de especialización, no del modelo arquetípico. De hecho, es lo más alejado de éste. La sofisticación de las formas las distancia del simple arquetipo (Owen, 1847, pp. 49, 59). Pero, por relevantes que fueran estas "máscaras adaptativas", estos "disfraces" (Owen, 1847, p. 41, 81), de todos modos, no alcanzan a obstaculizar el diagnóstico de homologías o el reconocimiento del arquetipo en cualquier modificación. La pericia del anatomista se juzga por su eficiencia en distinguir esos vestigios. (Advirtamos nuevamente el carácter programático de la propuesta.)

Darwin sigue casi totalmente a Owen en esta intuición. La acción de la selección natural (la fuerza adaptativa) enmascara las huellas de comunidad de origen. Peor: al generar similitudes por convergencia, produce incluso falsas pistas.

Así, se mantiene la idea de una fuerza adaptativa que echa un velo sobre la unidad de tipo, cubriendo con la modificación de las partes, según objetivos funcionales específicos, las similitudes que prueban la pertenencia al mismo plan de organización.

Como dijimos, la diferencia está en el origen de la fuerza adaptativa, y en el poder distorsionador relativo que ésta tiene sobre la unidad de tipo. Otra vez, para Darwin, la fuerza adaptativa (la selección natural) podría prevalecer frente a la unidad de tipo, provocando en tal caso extremo la desaparición del patrón común. Al respecto, escribió:
Si suponemos que un progenitor temprano -llamémoslo el arquetipo- de todos los mamíferos, aves y reptiles, tuviera sus miembros construidos bajo el patrón general, para cualquiera que fuera el propósito para el que servían, podemos percibir la significación plena de la construcción homóloga de las extremidades a lo largo de toda la clase [...] Sin embargo, es concebible que el patrón general de un órgano pueda tornarse tan oscurecida hasta el punto de que finalmente desapareciera, por la reducción y al final completa remoción de ciertas partes, por la fusión de otras partes, y por la duplicación o multiplicación de otras [...] en las aletas de los gigantes y extintos saurios marinos [...] el patrón general parece así haberse parcialmente oscurecido. (Darwin, 1872, pp. 384385, énfasis nuestro)

Más allá de las diferencias que hemos apuntado, notemos la terminología: (1) escribe utilizando "arquetipo", y (2) menciona como ejemplo de distanciamiento de éste a la clase mamífero, en la cual el progenitor temprano (el arquetipo) está enmascarado, "oscurecido". Las mismísimas palabras que empleara Owen en 1843 y 1849.

\section{3. ÓRGANOS RUDIMENTARIOS/VESTIGIALES}

Owen sostiene que la presencia de órganos "rudimentarios" sólo puede ser explicada por la unidad de tipo (Owen, 1849, pp. 12, 15), pues no podría obedecer a razones adaptativas. Son porciones del arquetipo esencial no "aprovechadas" funcionalmente.

Darwin retoma ese rol para tales elementos, aunque traducido a su marco, cuando sostiene que su presencia no puede explicarse por la teoría de la selección natural, y sí por la teoría del origen en común ${ }^{9}$.Para ambos, estos órganos son la pista perfecta para encontrar homologías. Utilizando los casos de Owen (Owen, 1849, pp. 30-35), Darwin escribió:

Al buscar homologías de la misma parte en diferentes miembros de una clase, nada es más común, o más necesario, que el uso y descubrimiento de rudimentos. Este es bien conocido a partir de los dibujos de Owen sobre los huesos de las patas del caballo, el buey, y el rinoceronte. [...] En las obras de historia natural los órganos rudimentarios se dice que han sido creados en general "en nombre de la simetría", o con el fin de "completar el esquema de la naturaleza"; pero esto no me parece constituir expli- 
cación alguna, sino más bien la mención de un hecho. [...] Desde mi perspectiva de la descendencia con modificación, el origen de los órganos rudimentarios es simple. (Darwin, 1859, pp. 452-454)

Naturalmente, la importancia que revisten estos rasgos para la detección de homologías se traslada a la empresa clasificatoria (Darwin, 1859, pp. 455-456). Pero hay más.

Como vimos, Darwin sostiene que, para un creacionista, para quien las partes animales están prediseñadas para sus respectivas funciones, resulta anómalo encontrar órganos inútiles. En cambio, su presencia resulta una expectativa para su teoría evolutiva. Esta misma idea aparece en Owen en su ataque a los funcionalistas (Owen, 1849, p. 84), y probablemente, otra vez, Darwin la tomara de aquél.

Para Owen y su conformidad de tipo, es plenamente esperable la presencia de rudimentos e imperfecciones. Otra vez: lo que parece refutatorio para el teleologista, es confirmatorio para el enfoque oweniano, en el mismo sentido en que posteriormente lo será para el darwiniano.

Finalmente, existe un detalle nomenclatural. Si bien Darwin llama a estos órganos "rudimentos", como hiciera Owen, en un par de ocasiones los denomina "vestigios". "Rudimento" suena a algo que todavía resta desarrollarse/evolucionar, algo cuya función plena está en el futuro. "Vestigio", por su parte, suena a "reliquia", algo cuya funcionalidad está en el pasado. Pareciera aquí que la expresión "rudimento" refleja la convicción de Owen de que pueden potencialmente convertirse en brazos, patas, alas o pies (Owen, 1849, p. 65), mientras que para Darwin, si bien también están "disponibles" para un reclutamiento funcional posterior, "por ahora" constituyen sólo un "vestigio" de un ancestro de su portador. No obstante, también Owen veía a algunos órganos rudimentarios como reducciones de viejos rasgos plenamente adaptativos (Owen 1850, p. 16), esto es, como vestigios.

\section{CUESTIONES PROGRAMÁTICAS}

\subsection{El PAPEL De LA REDUNDANCIA}

Como vimos, para Owen el arquetipo es resultado de la multiplicación de una vértebra ideal de cuyos segmentos constituyentes los huesos vertebrados no son más que realizaciones o actualizaciones modificadas, algunas de ellas muy sofisticadas. La vértebra ideal se repite serialmente gracias a fuerzas estructurales que actúan en el embrión. Cuanto más simple el organismo, mayor la incidencia de estas fuerzas respecto de la adaptativa.

La iteración no sólo da la heurística de qué buscar (vértebras, o partes de vértebras, modificadas, en cualquier porción del esqueleto), sino que también muestra que la fuerza adaptativa destina distintas instanciaciones de la misma cosa a multiplicidad de funciones. Sin esa pluralidad de vértebras, no habría suficiente "material" sobre el cual pudieran conformarse las adaptaciones. La redundancia, en resumen, es lo que posibilita la asignación diferencial de formas específicas para funciones específicas.

La misma intuición tiene Darwin, aunque sin evocar la teoría de la vértebra:

También hemos visto antes que las partes que se repiten muchas veces están más sujetas al cambio, no sólo en número, sino en forma. Consecuentemente, tales partes, estando ya presentes en un número considerable, y siendo altamente variables, naturalmente proporcionan materiales para la adaptación a los propósitos más diferentes; aunque en general retendrían, a partir de la fuerza de la herencia, sendas huellas de su parecido fundamental $u$ original. (Darwin, 1872, p. 386)

Aunque reinterpretada en Darwin, la noción de redundancia se conserva.

\subsection{CASOS DE APLICACIÓN COINCIDENTES}

La coincidencia en los casos de aplicación de dos teorías rivales no es explicitada regularmente. Es curioso que así sea, porque, después de todo, la rivalidad está generada usualmente por una disputa sobre ámbitos de aplicación compartidos de modo que uno esperaría que este tipo de convergencias sea la regla más que la excepción siempre que "las regiones del mundo" a explicar por la teoría desplazante sea al menos parcialmente coincidente con las de la desplazada. Kuhn utiliza el lenguaje hansoniano de la carga teórica al escribir acerca de la inconmensurabilidad a través de la noción de cambio gestáltico, aunque generalmente la utiliza de manera metafórica, no siempre implicando lo que suele caracterizarse como inconmensurabilidad empírica. 
En nuestro caso, Darwin retiene casos de aplicación de la teoría arquetípica, como casos de aplicación de la teoría del origen en común. Esto permite decir, con cierta fiabilidad, que ésta conserva la capacidad explicativa de aquella teoría previa. Cuando Darwin piensa en sitios donde aplicar la teoría de comunidad de origen, incluye ejemplos que se pueden leer en Owen ( $y$ algunos también en Saint-Hilaire), incluyendo ejemplares o casos de aplicación exitosa paradigmáticos de dicha teoría ${ }^{10}$. Veamos algunos:

(a) Las mandíbulas de los insectos (Owen, 1843, p. 215; Darwin, 1872, p. 383)

(b) "Los sospechosos de siempre", con los que ilustra cómo los "incontables propósitos a las que las partes animales llamadas 'extremidades', [generan] la consecuente diversidad en sus formas y proporciones visibles" (Owen, 1849, p. 9): las extremidades anteriores en el dugón, el topo, el murciélago, el cuadrúpedo y el hombre. Todas ellas presentan unidad de tipo sin un correlato funcional (Owen, 1847, p. 127; 1849, pp. 3-9; Darwin, 1859, pp. 200, 434, 479; 1872, p. 383)

Otra vez, la teoría del origen en común de Darwin es rival de la teoría de los arquetipos de Owen. Esto logra facilitar, más que obstaculizar, el que todo el esfuerzo de encontrar casos de aplicación que Owen hace para la aceptación de su teoría de los arquetipos sea capitalizado por Darwin, sin pérdidas ni agregados. El campo de aplicación de la teoría de los arquetipos es el campo de aplicación de la teoría del origen en común.

\subsection{El VALOR DE LA UNIFICACIÓN}

Hemos dejado para el final un punto clave en esta consideración programática. Esta vez, y a diferencia de los otros casos, la coincidencia tal vez sí pueda deberse más a un clima de época que a una influencia directa ${ }^{11}$. Nos referimos a que más de veinte años antes de la aparición de OS -y en sincronía con las inquietudes de Darwin sobre el mismo tema-, Owen tenía en mente concretar un proyecto unificador que permitiera eclécticamente curar las heridas de la dicotomía unidad de tipo-condiciones de existencia que permeó el debate entre Saint-Hilaire y Cuvier en 1830.

En 1837, Owen escribe una carta a William Whewell en la que dice que el objetivo autoimpuesto en su tarea en anatomía comparada era la de formular "una teoría armoniosa que combine los enfoques transcendentalistas y teleológicos" (Fide Sloan, 1992, p. 43).
Amundson (2007) apunta correctamente que Owen incluso supone haber logrado esta mediación en $O n$ the Nature of Limbs. Dice Owen:

La comparación de los huesos de las extremidades está repleta de estas hermosas evidencias de diseño; pero nuestro propósito actual es reunir las indicaciones de que lo que a veces ha sido erróneamente considerado como el principio antitético, esto es, la unidad de plan que yace en el fondo de todas las modificaciones adaptativas. (Owen 1849, p. 34).

El esfuerzo de Owen tendrá un éxito sólo parcial. Por un lado, sí logra mostrar que no se trata de enfoques incompatibles sino perfectamente integrables a la historia natural. Como vimos anteriormente, las formas organísmicas están determinadas tanto por fuerzas estructurales conservadoras ("la relación entre los Vertebrata y el arquetipo") como por fuerzas adaptativas productoras de variantes ("el principio de adaptación [fitness] de las cosas") actuando en su oposición (Owen, 1849, p. 38). Ambas coexisten en la naturaleza, y forma, proporción y función, unidad de tipo y diversidad, son consecuencias de ellas.

Como hemos dicho, este esquema conciliador suma, en Owen, al creacionismo, en tanto que el creador del arquetipo conoce desde el comienzo todas las instanciaciones de sus metamorfosis (Owen, 1849, p. 71).

El reconocimiento de un Ejemplar ideal para los animales vertebrados prueba que el conocimiento de un ser como el Hombre debe haber existido antes de que el hombre apareciera. Porque la mente divina que planeó el Arquetipo también previó todas sus modificaciones. La idea arquetípica se manifestó en la carne bajo tal diversas modificaciones, sobre este planeta, mucho antes de la existencia de aquellas especies animales que realmente lo ejemplificaron. (Owen, 1849, pp. 85-86)

A su vez, y por otro lado, tanto la iteración vertebral como sus modificaciones adaptativas se deben ambas a causas secundarias internas actuando en la embriogénesis, aunque nunca las explicita: ${ }^{12}$

A qué leyes naturales o causas secundarias la sucesión y progresión ordenadas de tales fenómenos orgánicos pueden haber estado sujetas somos hoy día ignorantes. Pero si, sin la derogación del poder Divino, podemos concebir la existencia de tales ministerios, y los personificamos bajo el término "Naturaleza", nos damos cuenta de que desde la historia pasada de nues- 
tro globo ella ha estado avanzando con pasos lentos y señoriales, guiada por la luz arquetípica, en medio del naufragio de los mundos, a partir de la primera encarnación de la idea Vertebrata bajo su antigua vestimenta ictícola, hasta su despliegue en el glorioso traje de la forma Humana. (Owen, 1849, p. 86)

Owen prueba que se puede ser idealista -existe un plan en la mente de Dios- y no por eso adherir al fijismo (Amundson, 2005), en tanto que la forma sí cambia, sí evoluciona, y por razones naturales (Richards, 1987). Esta inclusión de causas secundarias para la diversificación involucra que Owen quiere aplicar un enfoque naturalista para el problema del origen de las especies (Amundson, 2005, p. 76), siempre con un acento en aspectos embriológicos más que ambientales, herencia esta de von Baer. De hecho, más tarde escribió que hacia 1850, él mismo había sostenido que las especies deben haber aparecido por "leyes naturales o causas secundarias" (Appel, 1987, p. 226) ${ }^{13}$. Con todo, nada de esto afectó su confianza en la providencia divina que lo planificó todo desde el principio.

Sin embargo, tanto su morfología idealista como sus vagas apelaciones a fuerzas adaptativas internas no resultaron del todo convincentes ${ }^{14}$, o, en el mejor de los casos, apenas pudieron instalarse cuando en 1859 irrumpen las más plausibles explicaciones darwinistas. Será entonces -y no antes- que se logra la reconciliación entre las partes del "gran debate en París". Pero, notemos, el plan de ambos era idéntico, y las soluciones, aunque distintas, están íntimamente conectadas. Esta conexión programática bien puede deberse a una preocupación de época, pero las numerosas afinidades que sucintamente hemos mencionado aquí difícilmente puedan conjuntamente ser atribuidas con justicia al azar. Por el contrario, Darwin adopta ex profeso muchas de las convicciones de Owen aprovechándolas argumentativamente en su favor (Appel, 1987, p. 230; Amundson, 2007), cuando no haciéndolas encajar como un todo en sus propios constructos teóricos, o modificándolas al punto de generar un cambio en nuestra visión del mundo de un calibre tal vez sin par en toda la historia de la ciencia.

\section{CONCLUSIONES}

Aunque en lo personal la relación entre Owen y Darwin experimentó en 1859 una inflexión, de una de colaboración, respeto y aun simpatía mutua, a una de enconada enemistad, es también entonces que todas las influencias del primero sobre el segundo quedan plasmadas. Aunque suela pensarse en ellos como sendos representantes de dos visiones del mundo distintas y en rivalidad, hemos mostrado que la nueva visión de mundo propuesta por Darwin se construyó sobre esfuerzos conceptuales previos aportados en gran medida por Owen, y en lo que fuera probablemente una de muchas otras influencias de parte del buen número de naturalistas de los cuales Darwin sin dudas se nutrió.

En un sentido, esto es inevitable. Por ejemplo, los autores revolucionarios no pueden inventar un nuevo lenguaje para comunicar sus nuevas ideas. En cambio, se ven obligados a expresarlas (y pensarlas) en los vehículos terminológicos antiguos y ya disponibles. Por eso es que Darwin utiliza los mismos términos que Owen para expresar ideas diferentes ("arquetipo", "unidad de tipo", "fitness"), modificando su significado, apelando a metáforas, analogías, y otras estrategias discursivas.

Pero la influencia es mucho más profunda. No es sólo el término "arquetipo" el que Darwin hereda de Owen, sino también los métodos y teorías que permiten construirlo. ¡Qué paso pequeño, convertir al arquetipo en un ancestro! Y, sin embargo, esta reinterpretación involucra el cambio más sustancial respecto de las tareas del biólogo, de la naturaleza de la ciencia misma, y, finalmente, de nuestro lugar en el mundo.

A su vez, hemos mostrado cuán heterogénea es esta influencia. El listado que -sin ánimos de exhaustividad- hemos comentado aquí, nos fuerza a tomar distancia del modo en que Kuhn presenta las revisiones abruptas supuestamente involucradas en las revoluciones científicas, una caracterización que ha llevado incluso a comparar maliciosamente la elaboración de un paradigma con la de un café instantáneo (Watkins, 1970, pp. 35-36). Sin embargo, cuando Kuhn se detiene a considerar estudios de caso específicos de estas etapas revolucionarias, los matices no tardan en aparecer, y es con este segundo enfoque con el que nos encontramos -al evaluar nuestro caso- identificados.

Creemos que repensar estas cuestiones contribuye a hacer justicia con Owen y con el genio de Darwin. La propuesta de Darwin consistió, al estilo de Newton, en tomarse en serio los avances de sus predecesores, muchos de ellos aparentemente incompatibles entre 
sí, armando un rompecabezas cuyas piezas, a primera vista, resultaban imposibles de encajar en un único cuadro coherente. No se trató de la generación espontánea de un nuevo paradigma. Sin embargo, esto no minimiza la ruptura que Darwin produjo con el mundo antiguo y su justificación trascendente, pero es un ejemplo que nos invita a sofisticar nuestras herramientas metateóricas para pensar el modo en que las ideas antiguas pueden reclutarse para nuevos fines, mostrando que en la historia de la ciencia, así como en la historia de la vida, la estructura se explica más por lo heredado, que por lo elaborado de cero para lidiar con el ambiente de turno. Este es el sentido es que puede plantearse, sin caer en la exageración de Dobzhansky (1973), la relevancia de la luz

\section{NOTAS}

1. Al inicio de su carrera, Owen defendió el funcionalismo gracias a la influencia temprana de Cuvier. Poco a poco fue inclinándose -ya sin retorno- hacia el estructuralismo. (Appel, 1987; Amundson, 2005; Rupke, 2009a).

2. Escribe también de "homologías seriales" (simetría bilateral en el mismo individuo), y de la hipótesis de "homología general", sobre la cual nos detendremos luego. En lo sucesivo, cuando empleamos "homologías", a secas, nos estamos refiriendo siempre a las especiales.

3. Además del criterio topográfico, Owen también alude al histológico y al ontogenético.

4. Notemos cómo el reconocimiento de homologías precede tanto a su explicación histórica como a su explicación trascendentalista. Ambas teorías son rivales, justamente porque sus respectivos conjuntos de aplicaciones pretendidas son coextensivos entre sí, o al menos de intersección no vacía (Díez, 2012).

5. Las traducciones de todas las citas de textos referenciados en su versión original inglesa es nuestra.

6. Con todo, cuando explicita un grupo contemporáneo cercano al arquetipo, Owen menciona repetitivamente al lepidosirena, y no al anfioxo (Owen, 1849, p. 82). La correspondencia del arquetipo no sólo se daba con las formas vertebradas más simples (que es lo que enfatizamos aquí), que la historia de la ciencia arroja sobre la filosofía de la ciencia, análogamente con la de la teoría de la evolución orgánica sobre la biología.

\section{AGRADECIMIENTOS}

Este trabajo fue realizado con el financiamiento de PUNQ 1401/15 (Universidad Nacional de Quilmes, Argentina), UNTREF 32/15 255 (Universidad Nacional Tres de Febrero, Argentina), PICT-2014-1741 (ANPCyT, Argentina), PICT 2018-3454 (ANPCyT, Argentina) y UBACYT 20020170200106BA (Universidad de Buenos Aires, Argentina).

sino también con la fase más temprana -menos diferenciada- del embrión.

7. Estos tipos están fundamentados por razones funcionales en Cuvier. En von Baer y Owen, la justificación es estructural. Curiosamente, las categorías son las mismas. (Amundson, 2005, p. 42).

8. En las últimas ediciones de OS, Darwin aplaude el esfuerzo reconstructivo de Ernst Haeckel (1866) quien propone un único árbol genealógico.

9. De hecho, existe una tensión aquí: similitudes adaptativas, se explican gracias a la teoría de la selección natural; mientras que similitudes que involucran órganos inútiles, se explican gracias a la teoría del origen en común (Sober, 2011).

10. Owen menciona también los rasgos de Draco volans como ilustración tanto de homología como de analogía (Owen, 1843, p. 198; 1847, p. 7; 1849, pp. 45,82 ). Éste, si bien no es mencionado por Darwin en OS, sí lo es frecuentemente en libros de textos modernos y en museos de historia natural.

11. Otro autor que procuró lo mismo fue Milne Edwards, cuyo estudio soslayamos.

12. En 1859 , todavía consideraba al origen de las especies como un misterio incluso mayor que el de la extinción (Owen, 1859, p. 55).

13. Esto justifica su enojo cuando Darwin (1859, p. 310) lo tilda de un "vehemente" creyente en la inmutabilidad de las especies. Darwin enmendó 
esto posteriormente con su Historical Sketch (Owen 1866-1868, vol. 3, pp. 796-800; 1850, p. 15; Johnson, 2007; 2018; Bowler 2013). Para la década de 1860, Owen (1866-1888, vol. 3, p. 808) aceptaría claramente la teoría de la "derivación", esto es "que las especies cambian con el tiempo". Su escepticismo para con la teoría de la selección natural, su búsqueda de aspectos singulares en el

\section{BIBLIOGRAFÍA}

Aboitiz, Francisco (1988), "Homology: A comparative or a Historical Concept?", Acta Biotheoretica. 37, pp. 27-29.

Amundson, Ron (1998), "Typology reconsidered: Two doctrines on the history of evolutionary biology", Biology and Philosophy, 13, pp. 153-177.

Amundson, Ron (2005), The Changing Role of the Embryo in Evolutionary Thought, Cambridge, Cambridge University Press.

Amundson, Ron (2007), "Richard Owen and Animal Form". En: Amundson, Ron (ed.), Richard Owen. On the Nature of Limbs, Chicago, The Chicago University Press, pp. xv-li.

Appel, Toby (1987), The Cuvier-Geoffroy Debate: French Biology in the Decades Before Darwin., Oxford, Oxford University Press

Asma, Stephen (1996), Following Form and Function, Evanston, Northwestern University Press.

Blanco, Daniel (2011), "La teología natural y los "hechos" de la teoría evolutiva". En: Stefano, Waldir y Magda Medhat Pechliye (eds.), Filosofia e História da Biologia, Sâo Pablo, Mack Pesquisa, pp. 7-44.

Blanco, Daniel (2012), "Primera aproximación estructuralista a la teoría del origen en común", Agora, 31, pp. 171-194.

Bowler, David (2013), Darwin Deleted, Chicago, The Chicago University Press.

Darwin, Charles (1851), A Monograph of the Sub-class Cirripedia, vol. 1. Londres: The Ray Society.

Darwin, Charles (1859), On the Origin of Species, Londres, John Murray. $6^{\text {th }}$ ed. 1872.

Dawkins, Richard (2006), The Blind Watchmaker, Nueva York, Norton.

Díez, José (2012), "Inconmensurabilidad, comparabilidad empírica y escenas observacionales". En: Lorenzano, Pablo y Oscar Nudler (eds.), El camino desde Kuhn, Madrid, Biblioteca Nueva, pp. 67-118

Dobzhansky, Theodosius (1973), "Nothing in Biology Makes Sense except in the Light of Evolution", American Biology Teacher, 35, pp. 125-129.

Goodsir, John (1844), “On the anatomy of Amphioxus lanceolatus; Lancelet, Yarrell", Transactions of the Edinburgh Royal Society, 15, pp. 247-264. hombre, y su confianza en el poder creador de fuerzas internas continuaron hasta el final.

14. Amundson (2007) subraya que en Archetype (1847), es la fuerza adaptativa la que se encuentra permeada de platonismo, mientras que en Limbs (1849), esto sucede con el arquetipo. (También ofrece una explicación para este viraje, cuyo tratamiento excede a nuestros propósitos.).

Gould, Stephen (1978), “The Panda's Peculiar Thumb”, Natural Histor,. 87, pp. 20-30.

Gould, Stephen (1981), "Evolution as Fact and Theory", Discover, 2, pp. 34-37.

Griffiths, Paul (2007), "The phenomena of homology", Biology \& Philosoph,. 2, pp. 643-658.

Gross, Charles (1993a), “Hippocampus Minor and Man's Place in Nature: A Case Study in the Social Construction of Neuroanatomy", Hippocampus, 3, pp. 403-416.

Gross, Charles (1993b) "Huxley versus Owen: The hippocampus minor and evolution", Trends in Neuroscience, 16, pp. 493-498.

Haeckel, Ernst (1866), Generelle Morphologie der Organismen, Berlín, Georg Reimer.

Hull, David (1973), Darwin and His Critics. Chicago: University of Chicago Press,

Jacob, Francois (1977), "Evolution and Tinkering", Science, 196, pp. 1161-1166.

Johnson, Curtis (2007), "The Preface to Darwin's Origin of Species: The Curious History of the 'Historical Sketch'", Journal of the History of Biology, 40, pp. 529-556.

Johnson, Curtis (2018), "Charles Darwin, Richard Owen, and Natural Selection: A Question of Priority", Journal of the History of Biology, doi:10.1007/s10739-018-9514-2

Kuhn, Thomas (1970), The Structure of Scientific Revolutions, $2^{\circ}$ ed. Chicago, The Chicago University Press.

Laudan, Larry (1984), Science and Values, Berkeley, University of California Press.

Ochoa, Carlos; Ana Barahona (2009), Forma versus Función, México, UNAM.

Ospovat, Dov (1981), The Development of Darwin's Theory, Cambridge, Cambridge University Press.

Owen, Richard (1843), Lectures on Comparative Anatomy and Pysiology of the Invertebrate Animals, Londres, Longman.

Owen, Richard (1847), On the Archetype and Homologies of the Vertebrate Skeleton, Londres, Richard and John E. Taylor.

Owen, Richard (1849), On the Nature of Limbs, Londres, John Van Voorst. 
Owen, Richard (1850), “On Didornis (Part IV). Containing the Restoration of the Feet of that genus and of Palapteryx, with a Description of the Sternum in Palapteryx and Aptornis", The Transactions of the Zoological Society of London, 4, pp. 1-20.

Owen, Richard (1857), "On the Characters, Principles of Division, and Primary Groups of the Class Mammalia", Zoological Journal of the Linnean Society of London. 2, pp. 1-37.

Owen, Richard (1859), On the classification and geographical distribution of the Mammalia, being the lecture on Sir Robert Reade's foundation, delivered before the University of Cambridge, in the Senate-House, May 10, 1859. To which is added an appendix "on the gorilla," and "on the extinction and transmutation of species", Londres, John Parker.

Owen, Richard (1866-1868), On the Anatomy of Vertebrates, 3 vols, Londres, Longmans, Green, \& Co.

Padian, Kevin (2007), “Richard Owen's Quadrophenia”. En: Amundson, Ron (ed.), Richard Owen. On the Nature of Limbs, Chicago, The Chicago University Press, pp. liii-xci.

Paley, William (1802), Natural Theology, Londres, John Morgan.

Pearson, Christopher (2010), "Pattern cladism, homology, and theory-neutrality", History and Philosophy of the Life Sciences, 32, pp. 475-492.

Richards, Evelleen (1987), "A question of property rights: Richard Owen's evolutionism reassessed", British Journal of the History of Science, 20, pp. 129-171.

Roffé, Ariel (2020), "Dynamic Homology and Circularity in Cladistic Analysis", Biology \& Philosophy, Forthcoming.
Roffé, Ariel, Santiago Ginnobili; Daniel Blanco (2018), "Theoricity, Observation and Homology: A Response to Pearson", History and Philosophy of the Life Sciences. 40, pp. 1-8.

Rupke, Nicolaas (1993), “Richard Owen's Vertebrate Archetype", Isis, 84, pp. 231-251.

Rupke, Nicolaas (2009a), Richard Owen: Biology without Darwin, Chicago, The Chicago University Press.

Rupke, Nicolaas (2009b), "That the Theory of Organic Evolution Is Based on Circular Reasoning". En: Numbers, Ronald (ed.) Galileo Goes to Jail, Cambridge, Harvard University Press, pp. 131-141.

Russell, Edward (1916), Form and Function, Chicago, The Chicago University Press.

Sloan, Phillip (1992), "Introduction: On the edge of evolution". En: Sloan, Phillip (ed.), Richard Owen. The Hunterian Lectures in Comparative Anatomy, May and June 1837, Chicago, The Chicago University Press, pp. 3-72.

Sober, Elliott (2011), Did Darwin Write the Origin Backwards? Nueva York, Prometheus.

Watkins, John (1970), “Against 'Normal Science'”. En: Lakatos, Imre y Alan Musgrave (eds.), Criticism and the Growth of Knowledge, Cambridge, Cambridge University Press, pp. 25-38.

Wood, Stephen (1995), "The first use of the terms 'Homology' and 'Analogy' in the writings of Richard Owen", Archives of Natural History, 22, pp. 255-259. 